staffing constraints. Yusoff and colleagues have carried out a prospective study to determine whether the endoscopist performing the procedure can administer propofol safely and effectively.

Of 1,274 patients undergoing upper gastrointestinal EUS, 500 met selection criteria and were given moderate propofol sedation by the endoscopist performing the EUS procedure. Drug dosage, adverse events and complications were recorded. After the procedure, patient and endoscopist satisfaction levels were assessed using a questionnaire.

The mean propofol dose was $301 \mathrm{mg}$ (range 100-1000 mg). There were no EUSrelated complications or serious adverse events. Oxygen desaturation occurred in 16 (3\%) patients but was minor in 12 of these cases. Mild hypoxemia (saturation $>85 \%$ but $<90 \%)$ occurred in $4(1 \%)$ patients. No episodes of bradycardia, tachycardia or hypotension occurred. Patients who completed the questionnaire $(n=491)$ all reported that the procedure was 'very comfortable' and would elect to have the same sedation again. Endoscopists reported that the procedure was 'very smooth' or 'smooth' in 468 (94\%) cases and that administration of propofol was 'easy' in 451(90\%) cases.

The authors conclude that the administration of propofol by the endoscopist was safe and effective in these patients. They recommend, however, that the protocol be reserved for selected cases until more safety data are available.

Original article Yusoff IF et al. (2004) Endoscopist administered propofol for upper-GI EUS is safe and effective: a prospective study in 500 patients. Gastrointest Endosc 60: 356-360

\section{Early detection in gastric cancer}

Successful treatment in gastric cancer is limited by the late detection of tumors. A recent paper by Koike and colleagues compares two PCR-based screening methods for early detection.

Preoperative blood samples were obtained from 41 gastric cancer patients and 10 healthy volunteers. Samples were subjected to two assays: CEA-specific nested RT-PCR, in which a positive result indicates that circulating tumor cells are present in the original sample; and the MSP assay, which differentiates between methylated and unmethylated promoter regions of the p16, E-cadherin and RAR $\beta$ genes. Hypermethylation is a marker of downregulation or silencing of tumor suppressor genes, and hence indicates that tumor DNA fragments are present.

The CEA-specific RT-PCR assay gave a positive result in $10(24 \%)$ patients and the detection rate was higher in stage III or IV disease than in stages I or II $(P<0.01)$. The MSP assay detected aberrant methylation of p16 in $9(22 \%)$ patients, E-cadherin in 9 (22\%) patients and RAR $\beta$ in 6 (15\%) patients. Overall, 18 (44\%) patients showed aberrant methylation of the promoter region of at least one of the genes tested, and a positive result correlated with venous invasion $(P<0.05)$.

While the overall detection rate of the MSP assay was higher than that for CEAspecific RT-PCR, Koike et al. suggest that both assays will be useful for identifying patients requiring more intensive screening and treatment.

Original article Koike $\mathrm{H}$ et al. (2004) Comparison of methylation-specific polymerase chain reaction (MSP) with reverse transcriptase-polymerase chain reaction (RT-PCR) in peripheral blood of gastric cancer patients. J Surg Oncol 87: 182-186

\section{Albumin dialysis for acute-on- chronic liver failure}

MARS is an albumin-dialysis-based device that has been used to treat acute-on-chronic liver failure (ACLF). The treatment is based on the removal from the blood of proteinbound toxins, which might otherwise contribute to end-organ dysfunction. Since systemic inflammatory response is thought to be important in the pathophysiology of ACLF, Sen et al. have investigated whether and how MARS affects the plasma levels of cytokines, nitrate/nitrite (NOx), malondialdehyde (MDA) and ammonia.

Patients with alcoholic liver disease with ACLF were randomized to standard medical therapy (SMT; $n=9$ ) or SMT plus MARS $(n=9)$ over 7 days. Blood samples (collected at baseline, at 7 days and before and after each MARS treatment) were analyzed for

\author{
GLOSSARY \\ EUS \\ Endoscopic ultrasound \\ PCR \\ Polymerase chain reaction \\ CEA \\ Carcinoembryonic antigen \\ RT-PCR \\ Reverse transcriptase- \\ polymerase chain reaction \\ MSP \\ Methylation-specific PCR \\ RAR $\beta$ \\ Retinoic acid receptor- $\beta$ \\ MARS \\ Molecular adsorbents \\ recirculating system
}

